\title{
EVALUATING THE POTENTIAL OF DESIGN FOR ADDITIVE MANUFACTURING HEURISTIC CARDS TO STIMULATE NOVEL PRODUCT REDESIGNS
}

\author{
Alexandra Blösch-Paidosh \\ Engineering Design and Computing Lab \\ Department of Mechanical Engineering \\ ETH Zürich \\ Zürich, Switzerland
}

\author{
Prof. Saeema Ahmed-Kristensen \\ Design Products \\ School of Design \\ Royal College of Art \\ London, UK
}

\author{
Prof. Kristina Shea \\ Engineering Design and Computing Lab \\ Department of Mechanical Engineering \\ ETH Zürich \\ Zürich, Switzerland
}

\begin{abstract}
Additive manufacturing (AM) affords those who wield it correctly the benefits of shape, material, hierarchical, and functional complexity. However, many engineers and designers lack the training and experience necessary to take full advantage of these benefits. They require training, tools, and methods to assist them in gaining the enhanced design freedom made possible by additive manufacturing. This work, which is an extension of the authors' previous work, explores if design heuristics for AM, presented in a card-based format, are an effective mechanism for helping designers achieve the design freedoms enabled by AM. The effectiveness of these design heuristic cards is demonstrated in an experiment with 27 product design students, by showing that there is an increase in the number of unique capabilities of $A M$ being utilized, an increase in the AM novelty, and an increase in the AM flexibility of the generated concepts, when given access to the cards. Additionally, similar to the previous work, an increase in the number of interpreted heuristics and AM modifications present in the participants' designs when they are provided with the heuristic cards is shown. Comparisons are also made between 8-heuristic and 29-heuristic experiments, but no conclusive statements regarding these comparisons can be drawn. Further user studies are planned to confirm the efficacy of this format at enhancing the design freedoms achieved in group and team design scenarios.
\end{abstract}

\section{INTRODUCTION}

Additive manufacturing (AM) affords those who wield it correctly the benefits of shape, material, hierarchical, and functional complexity [1]. These unique capabilities have sparked the interest of designers and engineers the world over, many of which are eager to incorporate the benefits of AM into their designs. However, they lack the training and experience regarding the processes' capabilities and limitations that exist for other more traditional forms of manufacturing. As a result, prospective AM designers and engineers require training, tools, and methods to assist them in their new ventures and to help them break out of their traditional manufacturing mindsets [2].

In response to this need, many researchers, hobbyists, and industry workers are developing design for additive manufacturing (DfAM) methods and aids to address these training gaps. These DfAM methods and aids fall broadly into two categories: opportunistic and restrictive DfAM [3]. Restrictive methods consist mainly of design and printing guidelines (e.g. $[4,5])$ and are predominately useful in the later phases of the product development process [6], such as Detailed Design and Production Ramp-Up [7]. On the other hand, the opportunistic methods assist engineers and designers in expanding the limits of their knowledge with regard to AM and improving their design to take better advantage of the unique capabilities that AM affords them. Prior to the past few years, the development of DfAM methods has been dominated by restrictive and opportunistic topology optimization methods [8- 
10]. However, recently there has been a lot of interest in developing opportunistic DfAM methods that help people during the Conceptual Design phase [11-16], including previous work by the authors in developing design heuristics for additive manufacturing derived from a synthesis of the key AM features of 275 AM artifacts sourced from academia, industry, the media, and hobbyists [15]. These heuristics have already been validated, but they lack a fixed delivery format and their ability to stimulate the inclusion of the four unique capabilities of AM and increase the AM novelty and AM flexibility of the designs has not been evaluated. Therefore, as an extension of this previous work, this paper presents design heuristic cards that correspond to each of the previously developed design heuristics for AM and determines if these design heuristics for AM, presented in a cardbased format, are an effective mechanism for helping designers achieve the design freedoms enabled by AM. This is measured through the unique capabilities of $A M$ found in designs and the effect they have on the AM flexibility, AM novelty, and fluency of the design solutions.

This paper first offers background information on relevant topics before presenting the design heuristics for additive manufacturing cards and describing the experiment and analysis procedure in the method section. Next, the results of the experiment are reported and discussed. Finally, the paper concludes with a summary and discussion of future work.

\section{BACKGROUND}

It has been found that designers find opportunistic additive manufacturing knowledge (AMK) much more useful than restrictive AMK in the early phases of the design process [7]. With this in mind, several research groups have been working on tackling the transfer of opportunistic AMK to designers in the early phases of the design process. For example, [14] looked at using a small AMK booklet presenting ten AM potentials with the goal of stimulating radical and architectural innovation, and [11] investigated combining existing, established design methods with AMK to assist designers with DfAM in the early phases of the design process. [12] provided information sheets about various AM concepts to designers during the Conceptual Design phase, and [16] explored the use of Mood Boards for visual inspiration, a checklist of the most important AM design rules, an interactive system for AM design potentials, and 3D models for AM design potentials. Similar to the authors of this paper, [13] developed a list of design principles for AM based on the analysis of a quantity of artifacts from the hobby AM website, Thingiverse ${ }^{1}$. Unlike the design heuristics for AM derived by the authors of this paper, in [15], the design principles included both opportunistic and restrictive principles.

One thing that all these methods have in common is that they all use text to describe each principle/heuristic/potential. Most of the methods also combine these descriptive texts with images in the delivery format. There has also been some investigation into what elements users find functional, practical, and easy to understand when it comes to DfAM aids [7]. They asked designers to evaluate four different formats: text, physical artifacts, videos, and pictures. Videos, artifacts, and pictures were all highly rated by the participants (5-point Likert scale), but opinions were mixed regarding the text-based formats. However, they did not investigate any combined formats. In the end, they chose to build a video- and picture-based AMK tool, which also includes limited text, presumably, because it is not possible to fully explain concepts and transfer standard nomenclature without at least some text.

All of the previously mentioned studies used some form of verification or validation of their methods. This ranged from comparison with literature [13] to user surveys [7, 11, 16] to third-party evaluation of the designs generated in controlled user studies $[12,14,15]$. Those that used user surveys mainly focused on the opinions of the users as to whether or not they thought the method was useful or easy to use. However, in such cases, objective evaluation of the method in terms of key performance indicators was missing. Several studies examined the effect of the previous AM knowledge of the participant on the assessment of the method $[12,16]$. Additionally, almost all of these studies mentioned the importance of the innovative effect that incorporating AM into the early phases of the design process can have. However, only one of these studies objectively assessed this effect [14], and none of the studies looked at the effect of the method on the prevalence of the four unique capabilities of AM in the results, namely shape, material, hierarchical, and functional complexity [1].

Based on the above-mentioned AMK transfer methods, we propose to develop physical cards to accompany each of the previously derived design heuristics for AM, containing a mixture of both text and images so that the relevant AMK is fully transferred and easy to understand. Their ability to effectively assist designers in achieving the design freedoms enabled by AM must also be evaluated, because an AMK transfer method must be shown to objectively and effectively transfer AMK in a controlled experiment and not just receive positive feedback from the users, before it can be deemed a worthwhile tool for designers.

\section{METHOD}

Design Heuristic Cards for Additive Manufacturing

As previously stated, physical design heuristics for AM cards are developed that use both text and images to convey the relevant content to the user. Each card contains seven different pieces of information:

1. Design Heuristic

2. Design Heuristic Number

3. Description of the Design Heuristic

4. Design Heuristic Category

5. Image of an Abstract Example

6. Image of an Example from Industry or Literature

7. Short Description of the Real-World Example An example card with each of these pieces of information labelled is available in Figure 1.

\footnotetext{
${ }^{1}$ https://www.thingiverse.com/
} 
Table 1: Categorized Design Heuristics for AM for which Cards are designed. Superscript numbers indicate to which card set each heuristic belongs. "E" indicates the example card.

\section{Part Consolidation \\ 1 Consolidate parts for better functional performance ${ }^{3}$ \\ 2 Consolidate parts to reduce assembly time $^{3}$ \\ 3 Consolidate parts to increase robustness ${ }^{2}$ \\ 4 Consolidate parts to achieve multiple functions ${ }^{1}$}

\section{Customization}

5 Customize geometry to use case ${ }^{1}$

6 Customize user interface to use case $\mathrm{e}^{3}$

7 Customize artifact with decoration ${ }^{2}$

\section{Convey Information}

8 Convey information with color ${ }^{1}$

9 Convey information with geometry ${ }^{3}$

10 Convey information with haptics ${ }^{2}$

11 Convey information with light ${ }^{\mathrm{E}}$

Material

\section{Material Distribution}

15 Absorb energy with small interconnected parts ${ }^{1}$

16 Allow movement with small interconnected parts ${ }^{1}$

17 Use material distribution to achieve desired behavior ${ }^{2}$

18 Remove material to provide function ${ }^{2}$

19 Optimize structural topology or geometry ${ }^{3}$

Embed-Enclose

20 Embed functional material $^{3}$

21 Embed functional component $^{1}$

22 Use enclosed, functional parts ${ }^{2}$

Lightweight

23 Replace internal structure with lightweight lattice structure ${ }^{3}$

24 Hollow out artifact to reduce weight ${ }^{2}$

Reconfiguration

25 Create multi-functional artifact with reconfigurable structures ${ }^{1}$

\section{Use single material to achieve recutelability}

Recycling at the end of the product lifecycle can often be a challenge because of the mix of materials present in artificts. However metamaterials a $\mathbf{3}$ pecial material distributions aliow the achievement of unique and variable material properties while only using a single construction material throughout the part, which eases end-of-life recycling.

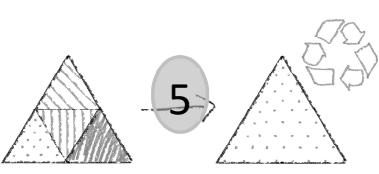
example, but the real-world example solutions on the back help them understand the heuristic in a real context. Additionally, because the examples are sketched instead of being photographs, the relevant parts of the example can be highlighted and distracting aspects can be lowlighted. However, one aspect of those cards that has proved problematic is that they are not categorized by the relevant design phase, something that makes using them with novices difficult because novices sometimes like to get ahead of themselves while designing. As the design heuristics for AM are largely relevant in the early phases of the design process, the need is not seen to classify them by design phase, but they are categorized into similar groups, so as to assist the user in evaluating multiple possibilities for solving the same problem or providing them with different but related heuristics.

Following the previous work [15], all 29 heuristics are reexamined to eliminate duplicates, confirm their relevancy as a design heuristic for AM, and clarify their wording. Twice, two very similar heuristics are reduced to one, and two heuristics are eliminated. Table 1 contains the final list of the 25 design heuristics for which cards are created. They are each sorted into one of eight AM categories: part consolidation, customization, information communication, materials, material distribution, embed-enclose, lightweight, or reconfiguration. The full set of

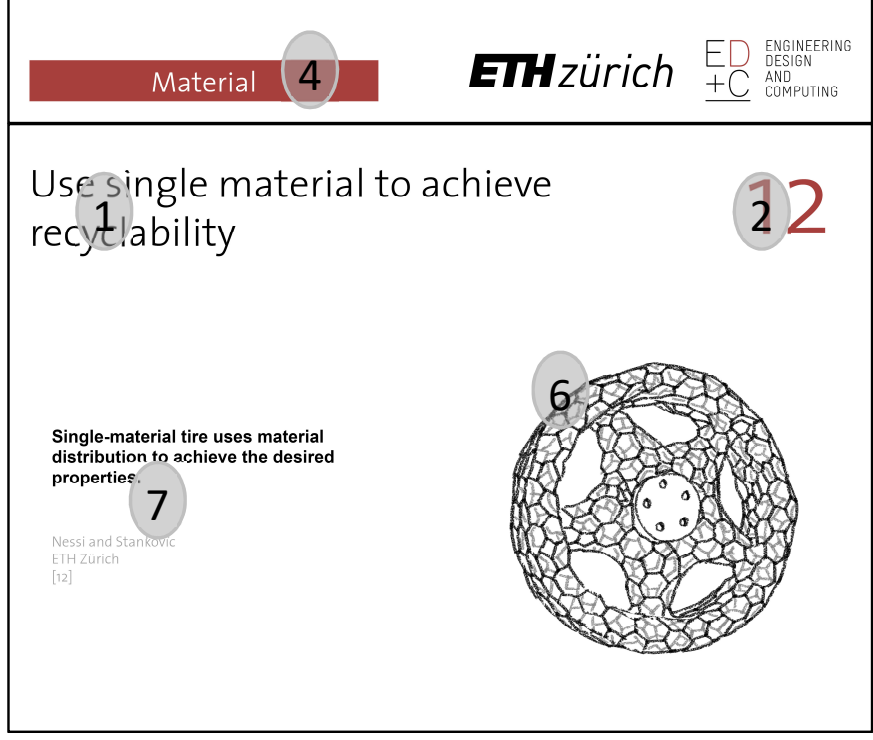

Figure 1. Example design heuristics for AM card. The front of the card is at the top and the back of the card is on the bottom. 
The company you are working for is looking at switching over from traditional manufacturing to additive manufacturing. To test out the idea, they would like you to redesign the Plantui to take advantage of the 4 unique capabilities of additive manufacturing (i.e. redesign it for additive manufacturing).

\section{Four Unique Capabilities of Additive Manufacturing}

- $\quad$ Shape complexity: ability to build almost any shape

- Hierarchical complexity: ability to build something with shape complexity across multiple size scales

- $\quad$ Functional complexity: functional artifacts can be produced in single or limited builds

- Material complexity: material is variable at every point in the build

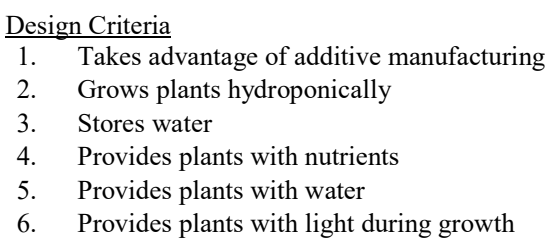

Please document only one concept per page. Clearly document your concept(s) with sketches and annotations so that they can be understood without you being there to explain it. Please write and sketch clearly. If you need more paper, please raise your hand.

At the end of this portion of the workshop, please place all your sheets of paper back in your envelope.

Figure 2. Design Task

cards is available for download from the lab's website ${ }^{2}$.

\section{Experiment Design}

After the development of the design heuristics for AM cards, their effectiveness at helping designers achieve the design freedoms enabled by AM must be assessed. This is accomplished via a controlled experiment with 27 product design master students at a UK University. Participation in the study is purely voluntary, and the participants are enticed to take part in the study by the opportunity to learn more about AM and DfAM. Each participant also receives a digital copy of the design heuristics for AM cards following the study. The students are not otherwise remunerated for their participation.

The experiment design is described below and additionally in Table 2. Prior to the start of the experiment, the participants are the recipients of an introductory lecture on DfAM and basic AM processes, and the unique capabilities of AM are explained to them. These topics are also interspersed with various AM examples from industry and literature. They are also introduced to the concept of design heuristics and how to use them, and the various portions of the design heuristics for AM cards are explained to them using one of the 25 cards. Following the lecture, they were instructed to fill out an AM Experience Survey, which surveyed their general design skills and DfAM

\footnotetext{
${ }^{2} \mathrm{http}: / /$ www.edac.ethz.ch/Research/Design-Heurestics-AM.html

${ }^{3}$ Only 24 of the 27 participants successfully and completely filled-out the electronic pre-survey. For this reason, when cross-referencing survey data during the analysis, the n-values are lower than for the experimental data.
}

Table 2. Experiment Design

\begin{tabular}{l|cc}
\multirow{2}{*}{ Order of Activites } & \multicolumn{2}{|c}{ Group } \\
\cline { 2 - 3 } & $\begin{array}{c}\text { Control } \\
\mathrm{n}=15\end{array}$ & $\begin{array}{c}\text { Experiment } \\
\mathrm{n}=12\end{array}$ \\
\hline $\begin{array}{l}\text { 1. Lecture on AM } \\
\text { (20 Min.) }\end{array}$ & $\mathrm{x}$ & $\mathrm{x}$ \\
\hline $\begin{array}{l}\text { 2. AM Experience Survey } \\
\text { 3. Recieves Design Heuristics } \\
\text { for AM Cards }\end{array}$ & $\mathrm{x}$ & $\mathrm{x}$ \\
\hline $\begin{array}{l}\text { 4. Completes Design Task } \\
\text { (45 Min.) }\end{array}$ & $\mathrm{x}$ & $\mathrm{x}$
\end{tabular}

skills and knowledge. It also surveys their degree of awareness of various DfAM topics (both opportunistic and restrictive) in the same manner as [3]. The awareness is surveyed using the same 6-point scale as [3], which is then converted into a linear scale for analysis ( 0 to 5$)$.

After the participants finish filling out the survey ${ }^{3}$, they are randomly split into two groups. The functions of the Plantui ${ }^{4}$, a small-scale, commercially available automatic, indoor, hydroponic urban farming device, are described to them during a live demonstration with the device. Then, they are each asked to redesign the Plantui for AM (see Figure 2 for the exact design task). The Plantui is chosen as the basis for the experiment for two reasons. First, it hits a good balance of simplicity and complexity, as it is a relatively small and simple system, but also has multiple functions and parts to which changes can be applied. Second, the authors have previously employed this case study successfully, and it thus allows comparison of data [15].

One group (Control group, 15 participants) receives the design task, an exploded view of the Plantui, a description of the function of each part, and some blank $\mathrm{A} 5^{5}$ pieces of paper on which to draw each of their concepts. The second group (Experimental group, 12 participants) receives everything the control group receives plus one subset of the design heuristics for AM cards. The cards are divided into three subsets of eight cards each. The cards are divided into three subsets because there is concern that the participants may suffer from an information overload for the allotted time if they are provided with all 25 cards, something the authors suspect happened in a previous, failed experiment. They are distributed in such a way so as to as equally as possible distribute the heuristics from each of the eight categories among the three subsets. The heuristic card that is used as the explanatory example is excluded from the study i.e. no subset receives this card. Care is taken during the distribution of the experiment materials to equally distribute the subsets of heuristic cards among the participants in the experimental group.

After the distribution of the materials, the participants are instructed that they have 45 minutes to complete the design task. They are also instructed to use one $\mathrm{A} 5^{5}$ page per concept, although they may include multiple AM capabilities in any one

\footnotetext{
${ }^{4}$ www.plantui.com

${ }^{5}$ A standard paper size in Europe
} 
concept. Finally, they are instructed that this is an individual task, and that all forms of communication with other participants or the outside world are prohibited during the experiment.

\section{Data Analysis}

The designs generated by the participants are evaluated using three different primary indices: interpreted heuristics, AM design modifications, and unique capabilities of AM. Some of these are later combined to derive secondary indices to help evaluate the effectiveness of the cards at enhancing the creativity of the generated designs, namely through AM novelty, design fluency, and AM flexibility. The index value interpreted heuristics is derived by analyzing each concept generated by a participant and determining which of the 25 design heuristics one could mine from the design. This is computed both cumulatively (i.e. the sum of all occurrences per concept) and count-wise (i.e. the number of different design heuristics applied per concept). The index value $A M$ design modifications is determined by counting the number of AM-relevant changes made to the original Plantui design. If the redesign is too radical to make connections between the design elements, it is excluded from evaluation of this, and only this, index value. Both of these indices were previously used by the authors during an earlier experiment and are derived in the same manner as in the previous work [15]. The final index value unique capabilities of $A M$, however, is new and is derived by evaluating each concept to determine each instance of occurrence of each of the four unique capabilities of AM as described by [1], namely shape, material, hierarchical, and functional complexity. This index value is also computed cumulatively and count-wise.

The AM novelty, or how unusually the design fulfills the design task with regard to AM, of each concept is assessed in two ways. First, the degree of AM novelty is assessed by summing together each occurrence of each interpreted heuristic and adding it to the sum of each occurrence of each unique capability of $A M$ for each concept. Second, the breadth of AM novelty is assessed by summing together a count of the number of different interpreted heuristics and a count of the number of different unique capabilities of AM for each concept. Designs that employ more heuristics and unique capabilities of AM are considered more novel from an AM perspective because they employ a wider range of AM applications.

The fluency, or the number of concepts produced in each group during the allotted time, is also evaluated. This is evaluated to determine if those using the design heuristic cards are less prolific in producing designs than those not using the cards.

To assess the AM flexibility of the concepts, or how well the designs are suited to fulfilling the design task through different AM means, the primary indices interpreted heuristics and unique capabilities of $A M$ are assessed at the group-level to determine if some heuristics or unique capabilities are only present in one group or the other. If, for example, some heuristics are only present in the experimental group, then it can be said that the heuristics help produce a more flexible array of designs. Designs that contain a wider variety of heuristics or capabilities are considered more AM-flexible because they employ AM in different ways and are therefore more likely to have a feasible AM implementation with the final, chosen AM process.

The designs are also evaluated at two different levels: participant and group. They are examined at the group-level so as to separate the individual participant from their concepts and instead be able to compare all of the work generated by the experiment group and compare it to the control group. When evaluated at the group-level, each sheet is considered one concept, and each concept is directly evaluated based on the above-discussed three different indices. Assuming that each participant creates at least one concept (i.e. one sheet), the sample size is at least as large as the number of participants in each group. However, when examined only at the group-level, any differences discovered in the pre-experiment AMknowledge survey cannot be controlled for during the analysis. Therefore, they are also examined at the participant-level. When examined at the participant-level, all of the index values generated for each concept associated with one participant are aggregated together, and the total number of concepts generated by that participant is noted.

Since two coders are not available to evaluate the entire data set, an intercoder reliability analysis is performed on a random sample $(10 \%)$ of the data for the index value unique capabilities of $A M$ using Cohen's Kappa [18] to show intercoder exchangeability. After the establishment of the intercoder exchangeability, the primary coder codes the remaining data. The kappa value for this index value is 0.871 , which is considered sufficient to establish intercoder exchangeability [19]. An intercoder reliability analysis is not performed for the other two primary indices because intercoder exchangeability for these indices was already established in previous work by the authors using the same coders and design task [15].

After the index values for each concept are determined and analyzed, the results are compared and contrasted to draw conclusions. All statistical operations are performed using SPSS.

\section{RESULTS}

The results comparing the three primary indices at both the group- and participant-level are visually summarized in Figure 3 and Figure 4, respectively. Throughout the analysis, at the participant-level, the primary indices are normalized by dividing the index value by the number of concepts generated by that participant. This is to prevent the particularly prolific designers from dominating the results. Due to space restrictions, only the summarized numerical results are presented here. The full data is available upon request to the authors.

It is found that access to the design heuristic cards affects the number of AM design modifications produced by participants at both the group- and participant-level. Those who have access to the cards produce more AM design modifications than those who do not have access to the cards. The same effect is seen on the other primary indices at both levels of analysis and both cumulatively and count-wise. Table 3 summarizes the relevant statistical data for each of these relationships according to the APA standard [20], namely including the degrees of freedom (df), 


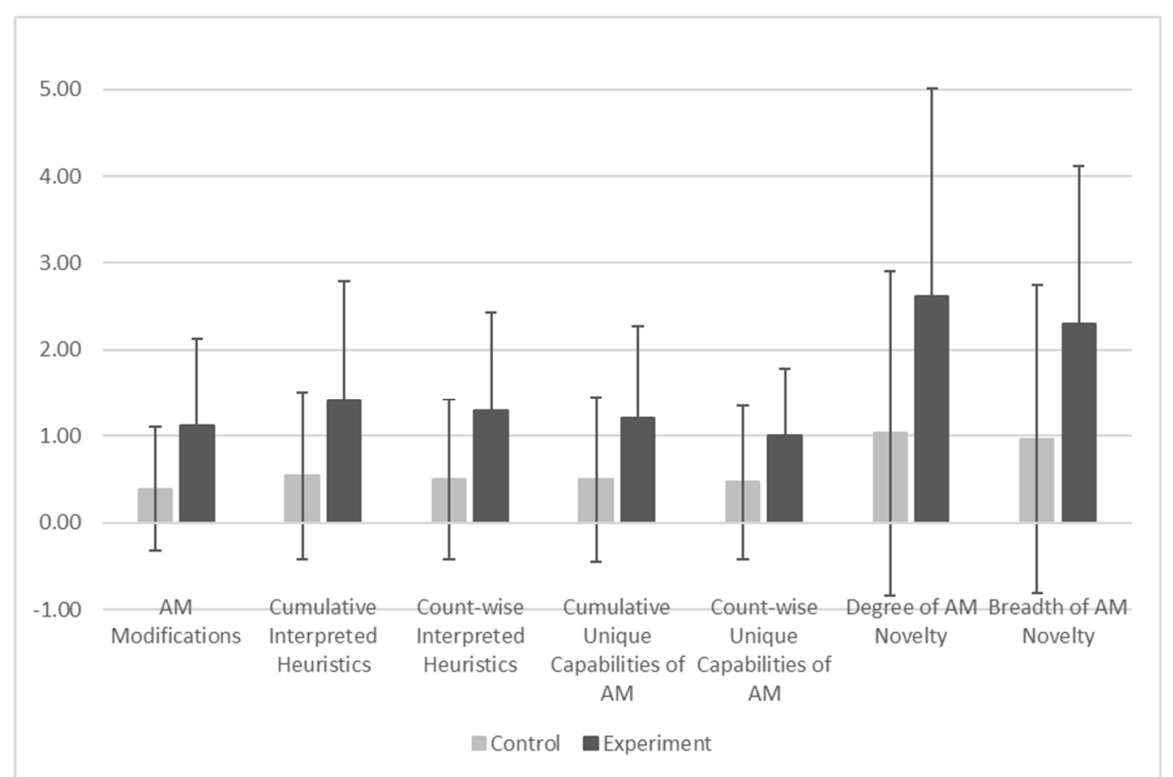

Figure 3. The means of various indices at the group-level. The error bars are $\pm 1 \mathrm{SD}$ of the mean. Control, $\mathrm{n}=54$. Experiment, $\mathrm{n}=34$.

t-value ( $(\mathrm{df}))$, the $p$-value $(\mathrm{p})$, and the effect size $(\mathrm{d})$. This is also the standard used for reporting the rest of the results in this paper.

At the participant-level, it is also found that there is a significant difference between the two groups' mean levels of self-reported AM design knowledge, although their mean levels of DfAM mean topic awareness show no significant difference (see Figure 4 and Table 3 for details). To determine if this difference in self-reported data affects the results, we run a MANCOVA with the self-reported AM design knowledge as the covariate. No significant effect is found $(\mathrm{p}>0.05)$.

The percentage of count-wise interpreted heuristics occurring in any given concept that belong to the same card-set as provided to the participant is analyzed in two MANOVAs (one for each analysis level, experiment group only). A significant effect is found at the group-level for the percentage of heuristics in Set $1\left(\mathrm{~F}(2,31)=11.54, \mathrm{p}<0.001, \eta^{2}=0.43\right)$, the percentage of heuristics in Set $2\left(\mathrm{~F}(2,31)=3.97, \mathrm{p}<0.03, \eta^{2}=.20\right)$, and the percentage of heuristics in Set $3\left(\mathrm{~F}(2,31)=39.62, \mathrm{p}<0.001, \eta^{2}\right.$ $=0.72$ ), but no significant effect is found at the participant-level $(\mathrm{p}>0.05)$. Tukey's post hoc is used to compare the differences between groups $(\mathrm{p}<0.05)$. The significant relations are available in Table 4, and Figure 5 visually summarizes the data. The indices indicated in Table 3 (plus the unique capabilities of AM broken down to the four categories) are also evaluated against

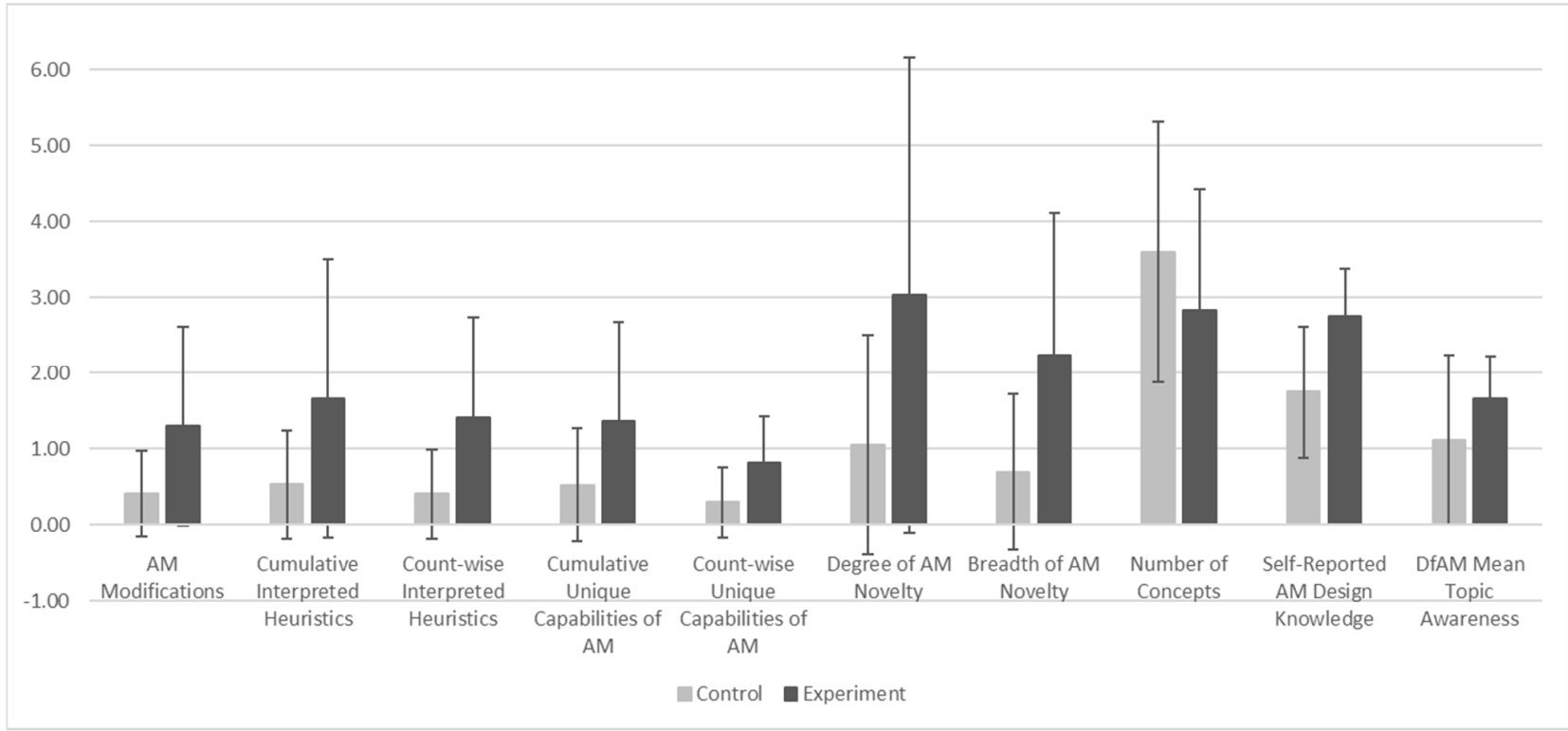

Figure 4. The means of various indices at the participant-level. The error bars are $\pm 1 \mathrm{SD}$ of the mean. Control, $\mathrm{n}=15$ (Except for self-reported AM design knowledge and mean AM awareness, $n=12$ ). Experiment, $n=12$. Except for the final three indices, the values are normalized by the number of concepts produced by the participants. 
Table 3. Statistical data for the mean comparisons. Non-significant relations are shaded.

\begin{tabular}{|c|c|c|c|c|c|c|c|c|}
\hline \multirow{2}{*}{ Index Value } & \multicolumn{4}{|c|}{ Group-Level } & \multicolumn{4}{|c|}{ Participant -Level (normalized) } \\
\hline & $\mathrm{df}$ & $t(d f)$ & $\mathrm{p}$ & $\mathrm{d}$ & $\mathrm{df}$ & $\mathrm{t}(\mathrm{df})$ & $\mathrm{p}$ & $\mathrm{d}$ \\
\hline AM Modifications & 86 & -3.97 & 0.000 & 0.87 & 25 & -2.37 & 0.026 & 0.92 \\
\hline Cumulative Interpreted Heuristics & 86 & -3.51 & 0.001 & 0.77 & 25 & -2.20 & 0.037 & 0.85 \\
\hline Count-wise Interpreted Heuristics & 86 & -3.57 & 0.001 & 0.78 & 25 & -2.67 & 0.013 & 1.03 \\
\hline Cumulative Unique Capabilities of AM & 86 & -3.24 & 0.002 & 0.71 & 25 & -2.11 & 0.045 & 0.82 \\
\hline Count-wise Unique Capabilities of AM & 86 & -2.90 & 0.005 & 0.64 & 25 & -2.58 & 0.016 & 1.00 \\
\hline Degree of AM Novelty & 86 & -3.46 & 0.001 & 0.76 & 25 & -2.17 & 0.039 & 0.84 \\
\hline Breadth of AM Novelty & 86 & -3.39 & 0.001 & 0.74 & 25 & -2.72 & 0.012 & 1.05 \\
\hline Number of Concepts & - & - & - & - & 25 & 1.19 & 0.246 & 0.46 \\
\hline Self-Reported AM Design Knowledge & - & - & - & - & 22 & -3.25 & 0.004 & 1.33 \\
\hline Mean AM Awareness & - & - & - & - & 22 & -1.90 & 0.071 & 0.78 \\
\hline
\end{tabular}

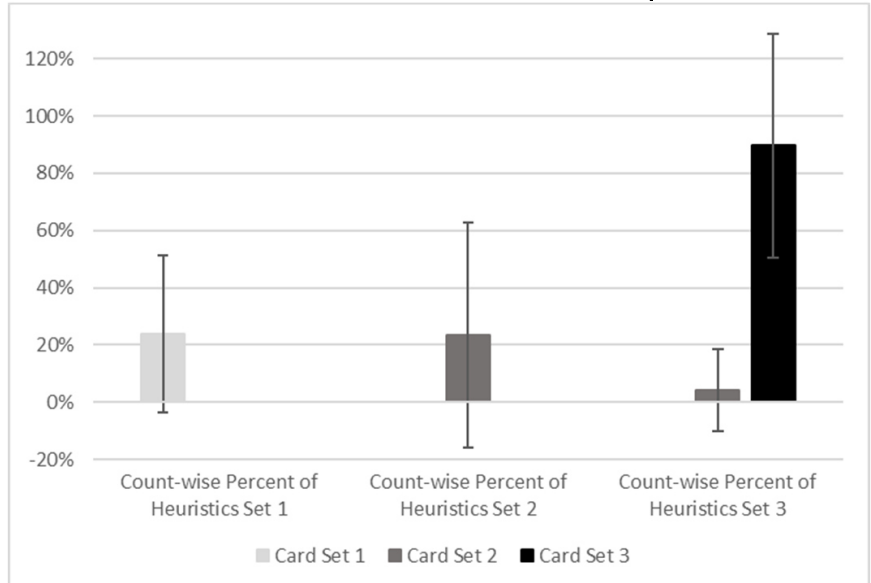

Figure 5. Compares the means of the percentage of heuristics per set at the group-level. The error bars are \pm 1 SD of the mean. Card Set $1, n=$ 6. Card Set 2, $n=12$. Card Set 3, $n=16$.

Table 4. Significant relations between card sets (group-level)

\begin{tabular}{c|c} 
Index Value & Significant Relation \\
\hline Count-wise Percent of Heuristics Set 1 & $\begin{array}{l}\text { Card Set } 1>\text { Card Set } 2 \\
\text { Card Set } 1>\text { Card Set } 2\end{array}$ \\
\hline Count-wise Percent of Heuristics Set 2 & Card Set $2>$ Card Set 3 \\
\hline Count-wise Percent of Heuristics Set 3 & $\begin{array}{l}\text { Card Set } 3>\text { Card Set } 1 \\
\text { Card Set } 3>\text { Card Set } 2\end{array}$
\end{tabular}

the different sets of heuristic cards in a MANOVA at the grouplevel, but no significant relations are found ( $p>0.05)$.

The relationship between the relevant interpreted heuristics and the participants' self-reported awareness of certain DfAM topics is analyzed to determine if there is any correlation between the number of occurrences of the relevant heuristics and the participants' existing knowledge. No correlations are found.

The effect of the cards on the creativity of the designs through enhanced design freedom is measured by AM flexibility, $A M$ novelty, and fluency. It is found that the pool of concepts generated by the experiment group has a higher degree of $A M$ flexibility than the concepts generated by the control group. The categories of customization and lightweight only occur in experimental group, and outside of these categories, heuristics \#3 Consolidate parts to increase robustness, \#12 Use single material to achieve recyclability, \#18 Remove material to provide function, \#19 Optimize structural topology or geometry, and \#21 Embed functional component only occur in the experimental group. Two heuristics only occur in the control group, \#11 Convey information with light and \#22 Use enclosed functional parts. The first served as the example heuristic in the pre-experiment brief, and the second occurs only once. In examining the unique capabilities of $A M$, no capability is present in only one group or the other, although the capabilities are more prevalent in the experiment group.

Next, in examining both measures of AM novelty, access to the design heuristics affects both of them at both analysis levels. In both cases, the measures of degree of AM novelty and breadth of AM novelty are higher when the participants have access to the design heuristics for AM cards. The relevant statistical information is in available in Figure 3, Figure 4, and Table 3.

Finally, the fluency of the two groups is also examined. The control group produces a total of 54 concepts and the experiment group 34. However, no significant difference is found between the mean number of concepts produced per participant in the control and experiment groups.

These results are compared, where possible, to those results from the authors' previous experiment with the design heuristics for AM [15]. This compares the use of 29 heuristics per person to 8 heuristics per person. There are significant differences between the control groups and the experiment groups. A visual summary of the differences is available in Figure 6 and the statistical data is available in Table 5. The 29-heuristic experiment produces higher levels of $A M$ modifications and interpreted heuristics, in both the control and experiment groups. However, the fluency of the 8-heuristic experiment is higher.

\section{DISCUSSION}

As the degree of AM novelty and the breadth of AM novelty increase in the presence of the heuristic cards, it can be 
Table 5. Statistical data for the inter-experiment mean comparisons at the participant-level (not normalized)

\begin{tabular}{|c|c|c|c|c|c|c|c|c|}
\hline \multirow{2}{*}{ Index Value } & \multicolumn{4}{|c|}{ Control } & \multicolumn{4}{|c|}{ Experiment } \\
\hline & $\mathrm{df}$ & $t(d f)$ & $\mathrm{p}$ & $\mathrm{d}$ & $\mathrm{df}$ & $\mathrm{t}(\mathrm{df})$ & $\mathrm{p}$ & $\mathrm{d}$ \\
\hline AM Modifications & 28 & 2.57 & 0.016 & 0.94 & 22 & 3.64 & 0.001 & 1.49 \\
\hline Cumulative Interpreted Heuristics & 28 & 2.26 & 0.032 & 0.82 & 19.32 & 4.49 & 0.000 & 1.77 \\
\hline Count-wise Interpreted Heuristics & 28 & 2.64 & 0.013 & 0.96 & 24 & 4.12 & 0.000 & 1.62 \\
\hline Number of Concepts & 14.00 & -5.84 & 0.000 & 2.13 & 11.00 & -4.00 & 0.002 & 1.58 \\
\hline
\end{tabular}

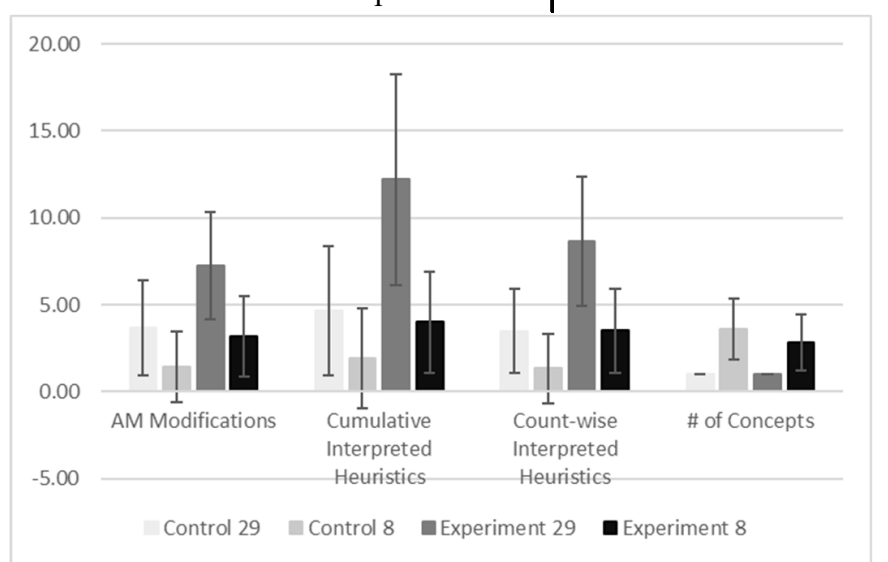

Figure 6. The means of various indices at the participant-level comparing a 29 heuristics experiment to a 8 heuristic experiment. The error bars are $\pm 1 \mathrm{SD}$ of the mean. Control 29, $\mathrm{n}=15$. Control $8, \mathrm{n}=$

15. Experiment 29, $\mathrm{n}=14$ (Except for AM modifications, $\mathrm{n}=12$ ). Experiment 8, $\mathrm{n}=12$.

concluded that the cards help produce concepts that are not only more novel from an AM perspective but are also employing a wider range of the novel aspects of AM. This is seen at both the group- and participant-level, which demonstrates that the effect is not isolated to single concepts, but also affects participants.

As all of the primary indices are higher when the participants have access to the cards, it can be concluded that the cards cause the users to increase the number of AM-relevant modifications that they make. Furthermore, they increase not only the number of heuristics present in their designs but also the number of unique capabilities of AM present in their designs, which indicates that use of the heuristic cards promotes not just the use of specific AM heuristics, but also promotes integration of the four unique capabilities of AM into the designs of AMnovices. As the increases are seen in both the cumulative and count-wise analyses, it can be seen that they are not only increasing the overall quantity but also employing a wider variety of heuristics and capabilities when using the cards. As this behavior is mirrored in both the group- and participant-level analyses, we see that this effect is seen not only in the groups as a whole, but also for individuals.

A significant difference in the self-reported $A M$ design knowledge level between the experiment groups is found, but it is found to have no significant effect on the results. Before the start of the experiment, participants are asked to assess their $A M$ design knowledge level as one of the following: none, fundamental awareness, novice, intermediate, advanced, or expert. As no context-specific definitions are provided for each of these levels, it is believed that this difference stems from different perceptions of the participants as to what each of these categories mean. Additionally, the survey does not specify if the knowledge is restrictive or opportunistic. As this design task focuses on the opportunistic aspects of AM, those who rate themselves higher in this respect may be thinking about their restrictive knowledge, which is most likely not very useful for this design task. To mitigate this, they are also asked to rate their awareness of several restrictive and opportunistic DfAM topics using a much more descriptive scale, which can then be aggregated to determine their DfAM mean topic awareness. The topics covered by the questionnaire skew more heavily toward opportunistic topics, so their mean DfAM topic awareness rating also skews toward their opportunistic DfAM awareness. This value is typically lower than their self-reported $A M$ design knowledge level and no significant difference between the mean topic awareness levels are found between the groups. Additionally, the individual topic awareness ratings are crossreferenced with the corresponding interpreted heuristics, and no significant correlations are found. For these three reasons, it is not believed that the prior knowledge of the participants has any effect on the results, and therefore the effects seen come solely from the use of the cards.

To add to the above conclusion, it is seen that the card set that the participant receives effects which interpreted heuristics are most likely to appear in their designs, namely those in the same heuristic card set, which also indicates that the effects seen are due to the cards and not due to the participants' knowledge. One can also assume that the three sets of cards equally promote AM modifications and each of the four unique capabilities of AM. There is no similar significant effect at the participant-level, but it is believed that this is due to the low sample sizes at the participant-level.

Related to this, the cards also lead to more flexible designs, as more heuristic categories and heuristics are present in the experiment group than in the control group. Similar to the previous related work [15], the categories of customization and lightweight only occur in the experimental group. These are categories, which those who are abreast of AM applications know are classic AM applications. Another classic application of AM that is only found in the experimental group is topology and geometry optimization. From this, one can conclude that the heuristic cards are helping the user come up-to-speed regarding AM applications.

A difference in fluency between the two groups is to be expected, as one group is not only designing, but also reading 
and using the cards. This is an effect also seen in other similar experiments $[17,21]$. However, it is surprising that there is no significant difference between the two groups when they are analyzed at the participant-level. This is believed to be due to a low sample size. However, the difference in fluency at the grouplevel may also partially stem from the difference in the number of participants in each group (15 in the control group vs. 12 in the experiment group).

Fluency differences are also observed between the 29- and 8-heuristic experiments. This is believed to be due to the type of participants in each experiment. The 29-heuristic experiment uses engineers-in-training [15] and the 8-heuristic experiment uses product designers-in-training. The engineers produced very detailed concepts containing many heuristics, but only one concept each. The product designers produced many more concepts on average, but most of them were much-less detailed, incorporating perhaps one or two heuristics. This could be a result of their respective trainings. However, in the case of the 29-heuristic experiment group, it is possible that the participants' fluency was hampered by the large number of heuristics they had at their disposal. More investigation of this phenomenon would need to be undertaken to make any definite statements.

The count-wise number of interpreted heuristics is also an interesting comparison between the two experiments. In the 29heuristics experiment, the most unique heuristics utilized by one participant is 15 of $29(52 \%)$. In the 8 -heuristics experiment, that number is 7 of $8(88 \%)$. This seems to indicate that reducing the number of heuristics supplied to the participants in a fixed-length design task may allow them to apply proportionally more of them. However, this conclusion is also clouded by the fact that, although similar in age, the participants of the two experiments have different training backgrounds and different levels of familiarity with the design task. A more focused study would need to be completed to draw any hard conclusions.

As evidenced by the above results and discussion, use of the heuristic cards by the participants leads to an overall increase in the enabled design freedom of AM. This is measured through the unique capabilities of $A M$, fluency, AM flexibility, and $A M$ novelty. Significant differences are found between the two experimental groups in three of the four metrics in favor of the experiment group, and these differences are shown to be a direct result of access to the cards and not the design skills or the existing AMK of the participants. Thus, one can conclude that the design heuristic for AM cards are an effective mechanism for helping designers achieve the design freedoms enabled by AM.

Following this experiment, the number and content of the heuristics are shown to be in a stable state. The cards also function effectively, and general feedback from the participants is neutral to positive about the cards in general and specific aspects of them. Interestingly, different people find different aspects of the card helpful. Some focus-in on the short text, others on the long text, and still others on the images and examples. This informal feedback indicates that the different aspects of the cards are helpful for different people, which indicates that the mix of text and images is a good decision. It may also be useful to add an additional example in the future. The multi-format aspect of the cards proved to be an asset to the users of the cards. Therefore, in the future, the design heuristics for AM cards will continued to be used in their current format.

One aspect of the cards that does not work so well is the size. They are designed to be printed on $\mathrm{A} 5^{6}$ paper ( $5.8 \times 8.3$ inches $)$, but it was found while observing the participants work with them, that they are large and clumsy in the hand, making it difficult to quickly tab through them or examine two at the same time (some of the particular benefits of using a physical-based system). Therefore, in the future, the size of the cards will be reduced, possibly to an $\mathrm{A} 6^{6}$ (4.1 x 5.8 inches).

The effect of the design heuristics for AM cards has been assessed in terms of both AMflexibility and AMnovelty, but there is one aspect of the heuristics' efficacy that has not yet been investigated: innovativeness. As this is a classic measure of design creativity, this is something that will be examined in the future.

Thus far, the effect of the design heuristics for AM has only been assessed during individual design scenarios. In the future, they will be studied in group- and team-design scenarios.

\section{CONCLUSION}

In this work, we present an extension of our previous work of developing design heuristics for AM [15]. The card-based format for communicating 25 previously derived design heuristics for $\mathrm{AM}$ is demonstrated to promote $\mathrm{AM}$ design freedom in an experiment with 27 product design students, by showing that there is an increase in the number of unique capabilities of $A M$ being utilized, an increase in the AM novelty, and an increase in the AM flexibility of the generated concepts, when given access to the cards. Additionally, similar to the previous work [15], an increase in the number of interpreted heuristics and AM modifications present in the participants' designs when they are provided with the heuristic cards is shown. Comparisons are also made between 8-heuristic and 29-heuristic experiments [15], but no conclusive statements regarding these comparisons can be drawn. Further user studies are planned to confirm the efficacy of this format at enhancing the design freedoms achieved in group and team design scenarios.

\section{ACKNOWLEDGMENTS}

The authors would like to thank those members of the Design Products Degree Program at the Royal College of Art (Autumn 2018) who participated in this experiment.

\section{REFERENCES}

[1] Gibson, I., Rosen, D., and Stucker, B., 2015, "Design for Additive Manufacturing," pp. 399-435. DOI: 10.1007/978-14939-2113-3 17

\footnotetext{
${ }^{6}$ A standard paper size in Europe
} 
[2] Seepersad, C. C., 2014, "Challenges and Opportunities in Design for Additive Manufacturing," 3D Printing and Additive Manufacturing, 1(1), pp. 10-13. DOI: 10.1089/3dp.2013.0006

[3] Prabhu, R., Miller, S. R., Simpson, T. W., and Meisel, N. A., 2018, "The Earlier the Better? Investigating the Importance of Timing on Effectiveness of Design for Additive Manufacturing Education," Proceedings of the ASME 2018 International Design Engineering Technical Conferences and Computers and Information in Engineering Conference (IDETC/CIE 2018))Québec City, Québec, Canada, 26-29 August 2018.

[4] Adam, G. A. O., and Zimmer, D., 2014, "Design for Additive Manufacturing - Element transitions and aggregated structures," CIRP Journal of Manufacturing Science and Technology, 7(1), pp. 20-28. DOI: 10.1016/j.cirpj.2013.10.001

[5] Stöckli, F., Modica, F., and Shea, K., 2016, "Designing passive dynamic walking robots for additive manufacture," Rapid Prototyping Journal, 22(5), pp. 842-847. DOI: 10.1108/rpj-11-2015-0170

[6] Ulrich, K. T., and Eppinger, S. D., 2008, Product Design and Development, McGraw Hill, Boston, Massachusetts, USA. ISBN: 978-007-125947-7

[7] Laverne, F., Segonds, F., D'Antonio, G., and Le Coq, M., 2016, "Enriching design with $\mathrm{X}$ through tailored additive manufacturing knowledge: a methodological proposal," International Journal on Interactive Design and Manufacturing (IJIDeM), 11(2), pp. 279-288. DOI: 10.1007/s12008-016-03147

[8] Seepersad, C. C., Allison, J., and Sharpe, C., 2017, "The Need for Effective Design Guides in Additive Manufacturing," In: Proceedings of the 21st International Conference on Engineering Design (ICED17), Vol. 5: Design for X, Design to X, Vancouver, Canada, 21-25 August 2017, pp. 309-316.

[9] Bourell, D. L., Rosen, D. W., and Leu, M. C., 2014, "The Roadmap for Additive Manufacturing and Its Impact," 3D Printing and Additive Manufacturing, 1(1), pp. 6-9. DOI: $10.1089 / 3 \mathrm{dp} .2013 .0002$

[10] Thompson, M. K., Moroni, G., Vaneker, T., Fadel, G., Campbell, R. I., Gibson, I., Bernard, A., Schulz, J., Graf, P., Ahuja, B., and Martina, F., 2016, "Design for Additive Manufacturing: Trends, opportunities, considerations, and constraints," CIRP Annals - Manufacturing Technology, 65(2), pp. $737-760$. DOI: $10.1016 /$ j.cirp.2016.05.004

[11] Watschke, H., Bavendiek, A.-K., Giannakos, A., and Vietor, T., 2017, "A Methodical Approach to Support Ideation for Additive Manufacturing in Design Education," In: Proceedings of the 21st International Conference on Engineering Design
(ICED17), Vol. 5: Design for X, Design to X, Vancouver, Canada, 21-25 August 2017, pp. 41-50.

[12] Laverne, F., Segonds, F., Anwer, N., and Le Coq, M., 2015 , "Assembly Based Methods to Support Product Innovation in Design for Additive Manufacturing: An Exploratory Case Study," Journal of Mechanical Design, 137(12), p. 121701. DOI: $10.1115 / 1.4031589$

[13] Perez, K. B., Anderson, D. S., Hölttä-Otto, K., and Wood, K. L., "Crowdsourced Design Principles for Leveraging the Capabilities of Additive Manufacturing," Proc. In: Proceedings of the 20th International Conference on Engineering Design (ICED15), Vol. 4: Design for X, Design to X, Milan, Italy, 27.30.07 .2014

[14] Yang, S., Page, T., and Zhao, Y. F., 2018, "Understanding the Role of Additive Manufacturing Knowledge in Stimulating Design Innovation for Novice Designers," Journal of Mechanical Design, 141(2). DOI: 10.1115/1.4041928

[15] Blösch-Paidosh, A., and Shea, K., 2019, "Design Heuristics for Additive Manufacturing Validated Through a User Study," Journal of Mechanical Design, 141(4). DOI: 10.1115/1.4041051

[16] Kumke, M., Watschke, H., Hartogh, P., Bavendiek, A.-K., and Vietor, T., 2017, "Methods and tools for identifying and leveraging additive manufacturing design potentials," International Journal on Interactive Design and Manufacturing (IJIDeM), 12(2), pp. 481-493. DOI: 10.1007/s12008-017-0399-

[17] Murphy, L., Daly, S., McKilligan, S., and Seifert, C. M., 2017, "Supporting Novice Engineers in Idea Generation using Design heuristics," 2017 Asee Annual Conference \& Exposition, ASEE, Columbus, Ohio, USA, p. 19008.

[18] Cohen, J., 1960, "A Coefficient of Agreement for Nominal Scales," Educational and Psychological Measurement, 20(1), pp. 37-46. DOI: 10.1177/001316446002000104

[19] Hayes, A. F., 2005, "Statistical Methods For Communication Science," Lawrence Erlbaum Associates, Inc., Mahwah, New Jersey, USA. ISBN: 9780805854879

[20] Schwartz, B. M., Wilson, J. H., and Goff, D. M., 2018, An EasyGuide to Research Design \& SPSS, SAGE Publications. ISBN: 9781506385464

[21] Keshwani, S., Lenau, T. A., Ahmed-Kristensen, S., and Chakrabarti, A., 2017, "Comparing novelty of designs from biological-inspiration with those from brainstorming," Journal of Engineering Design, 28(10-12), pp. 654-680. DOI: $10.1080 / 09544828.2017 .1393504$ 